\section{Microalbuminuria in stage 1 hypertension predicted by glomerular hyperfiltration}

In people with overt hypertension, microalbuminuria is associated with an increased incidence of fatal events. Detection of microalbuminuria should, therefore, trigger early intervention. But can the onset of microalbuminuria precede that of hypertension and, if so, how can its onset be predicted? Palatini et al. sought answers to these questions by analyzing data from the Hypertension and Ambulatory Recording Venetia Study (HARVEST).

Treatment-naive subjects with borderline (stage 1) hypertension but no microalbuminuria $(n=502)$ were followed for an average of almost 8 years. During follow-up, albumin excretion rate increased to a significantly greater extent in subjects with excessive glomerular filtration at baseline (creatinine clearance exceeding $150 \mathrm{ml} / \mathrm{min} / 1.73 \mathrm{~m}^{2}$ ) than in those without hyperfiltration $(P<0.001)$. When adjusted for confounding factors such as age, sex, $24 \mathrm{~h}$ blood pressure and $\mathrm{BMI}$, baseline creatinine clearance was found to be a strong independent predictor of the risk of subsequently developing microalbuminuria $(P<0.001)$. Interestingly, blood pressure measured in the clinic was not predictive of microalbuminuria.

According to current guidelines, which recommend that antihypertensive treatment is not initiated until blood pressure exceeds $140 / 90 \mathrm{mmHg}$, almost $50 \%$ of subjects in this study with glomerular hyperfiltration-and therefore an increased risk of microalbuminuria-did not require treatment. On the basis of their new data, the authors assert that people screened for stage 1 hypertension with glomerular hyperfiltration should be started on angiotensin-converting-enzyme inhibitors or angiotensin-receptor blockers even if their clinic-measured blood pressure spontaneously declines to within the normal range.

Original article Palatini P et al. (2006) Glomerular hyperfiltration predicts the development of microalbuminuria in stage 1 hypertension: the HARVEST. Kidney Int 70: 578-584

\section{Cystatin C level predicts heart failure in elderly black individuals with renal disease}

Chronic kidney disease increases the risk of heart failure. Both conditions are more likely to affect black individuals than whites. Researchers have used three common methods for measuring kidney function-serum creatinine level, plasma cystatin C level and estimated glomerular filtration rate (eGFR) using the Modification of Diet in Renal Disease equationto investigate whether the relationship between chronic kidney disease and incident heart failure differs between the two racial groups.

Among a subset of elderly participants from the Health, Aging, and Body Composition Study who had no self-reported history of heart failure $(1,124$ black and 1,676 white individuals aged between 70 and 79 years), 200 individuals developed heart failure over a mean period of 5.7 years. High cystatin $\mathrm{C}$ concentration and low eGFR were associated with heart failure in both black and white participants, but the association was much stronger in the former. The nearly linear association between heart failure and kidney dysfunction in black individuals as measured by cystatin $\mathrm{C}$, and this technique's good sensitivity at higher GFRs, indicate that cystatin $\mathrm{C}$ measurement is the most accurate means of assessing risk of heart failure in this racial group.

Elucidating the mechanisms responsible for the stronger association of kidney dysfunction with heart failure in black individuals requires further investigation. The authors suggest that there could be a risk factor common to kidney and heart disease, such as hypertension, that is more severe in those of black ethnic origin.

Original article Bibbins-Domingo K et al. (2006) Renal function and heart failure risk in older black and white individuals: the Health, Aging, and Body Composition Study. Arch Intern Med 166: 1396-1402

\section{Breast calcification in dialysis patients has a limited effect on mammogram interpretation}

The identification of certain types of calcification during breast cancer mammography screening can indicate malignant disease. It is well established that women receiving dialysis show an increased incidence of breast calcifications; however, data on the morphology of these calcifications are currently limited. This lack of data could make the interpretation of mammograms from dialysis patients more difficult, and cause unnecessary psychological stress as a result of erroneous callbacks. 